\section{Free lunch?}

SIR - The comprehensive review of the patterns and properties of food webs by Pimm et ll $^{1}{ }^{1}$ has been made possible by the recent compilation of large amounts of data on trophic relations in communities $^{2}$. But several unrealistic assumptions widespread in the literature block further insight into the principles of community energetics.

Pimm et al. follow ecological tradition and describe food webs as having a lower (or producer) level and an uppermost (or top predator) level. That there are producer organisms is a realistic postulate, although producers can be thought of simply as predators for sunlight and inorganic nutrients. That there are top predators, however, is not tenable, if by top predators we mean "species on which nothing else in the web feeds (page 670 of ref. 1). When a lion or an eagle dies, the matter of which it is composed (and the energy contained therein) does not simply disappear into the ether, nor does it pile up indefinitely, forming vast accumulations of predators' carcasses. Rather, these organisms are consumed by scavengers and detritivores. There is a general perception that these scavengers should not be included in food webs and trophic pyramids because they do not capture live organisms for consumption the way herbivores and predators do. But from the perspective of energy flow, the distinction between species that capture a prey item alive, kill it and then eat it, and species that consume organisms that are found dead is entirely artificial.

Food webs that do not draw artifical distinctions between the so-called top predators and the organisms that feed on them may affect our understanding of trophic patterns in several ways. For example, contrary to the claims of Pimm et al., trophic cycles are probably ubiquitous in food webs. When a top predator, such as an eagle, dies it may be eaten by scavenging flies, which might be fed on by insectivorous birds, which in turn may fall prey to an eagle, forming a cycle. In their Fig. 1, Pimm et al. depict various predators, parasites and hyperparasites as not having any species exploiting them; this is probably more a reflection of our ignorance of the natural history of many of these species than it is a biological fact. A more realistic representation of this figure would probably include arrows circling back into the web, forming trophic cycles - especially towards the upper parts of the food web. Cycles are probably not rare, but are perceived as such because of the unfortunate custom of leaving scavengers and detritivores out of the picture, and because of the scarcity of information about what feeds on the smaller organisms and on the rare predators and parasites. A more detailed knowledge of the natural history of biological communities may reveal that there are few, if any, species that are top predators in the sense of being consumers that do not become, in turn, any other species' lunch.

\section{Museum of Comparative Zoology}

Harvard University,

Cambridge,

Massachusetts 02138, USA

1. Pimm, S. L., Lawton, J. H. \& Cohen, J. E. Nature 350 669-674 (1991)

2. Cohen, J. E., Briand, F. \& Newman, C.M. Community Food Webs Data and Theory (Springer, New York, 1990)

SIR - Pimm et al. ${ }^{1}$ discuss community food web theory, based on the results of a previous survey involving analysis of 113 published studies ${ }^{2}$. But only $23 \%$ of these studies are from terrestrial ecosystems, the rest being mainly from submerged and semi-submerged habitats. Tropical rainforest ecosystems, containing most of the world's terrestrial species, are represented by only one study, previously described as incomplete ${ }^{3}$. Therefore, many of the general features of community food webs outlined by Pimm et al. may be more applicable to aquatic ecosystems than to many terrestrial ones.

The terrestrial detritus food web is a major component of most terrestrial ecosystems, and is directly responsible for regulating nutrient cycling, litter decomposition and energy flow. None of the 113 studies considered this food web in detail. Although terrestrial detritus food webs are relatively poorly understood, many of the features of food webs outlined by Pimm et al. do not seem to conform with the results of some recent studies on terrestrial detritus-based systems.

The following examples demonstrate this: (1) Omnivory is probably widespread in most detritus food webs. This does not apply only to organisms feeding on necromass (as noted by Pimm et al.), but also to several below-ground mite and nematode species which can feed on live organisms of more than one trophic level $^{4,5}$. (2) Within-habitat compartmentalization is a feature common to most detritus food webs. The two main saprophytic groups of organisms, bacteria and fungi, are morphologically quite distinct. Compartmentalization arises because most microbivores are either spe-

1. Pimm, S. L. Lawton, J. H. \& Cohen, J. E. Nature 350. 669-674 (1991)

2. Cohen, J. E., Briand, F. \& Newman, C. M. Community Food Webs: Data and Theory (Springer, New York, 1990).

3. Schoener, T. W. Ecology 70, 1559-1589 (1989).

4. Walter, D. E. Ecology 68, 226-229 (1987)

5. Walter, D. E., Hunt, H. W. \& Elliott, E. T. Pedobiology 31 247-260 (1988)

6. Hendrix, P. F. et al. Bioscience 36, 374-380 (1986).

7. Ingham. E. R. et al. J. appl. Ecol. 23, 597-614 (1986) cialized feeders on bacteria (protozoa and bacterivorous nematodes) or fungi (fungivorous mites and nematodes) ${ }^{6,7}$. There appears to be relatively little overlap between these compartments. (3) Many detritus food webs often contain more than four trophic levels ${ }^{7}$, probably as a result of the soil matrix serving as a highly buffered three-dimensional system.

Ruakura Agricultural Centre,

D. A. WARDLE

Private Bag,

Hamilton,

New Zealand

\section{Thin-film YBCO magnetometer}

SIR - There has recently been much progress in the development of circuits made from thin films of high-transitiontemperature $\left(T_{c}\right)$ superconductors. As a result, one can now construct sensitive magnetometers consisting of a d.c. SQUID (superconducting quantum interference device) coupled to a flux transformer to enhance its sensitivity to magnetic fields ${ }^{1}$. We have made ${ }^{2}$ a thinfilm $\mathrm{YBa}_{2} \mathrm{Cu}_{3} \mathrm{O}_{7-x}$ (YBCO) magneto-
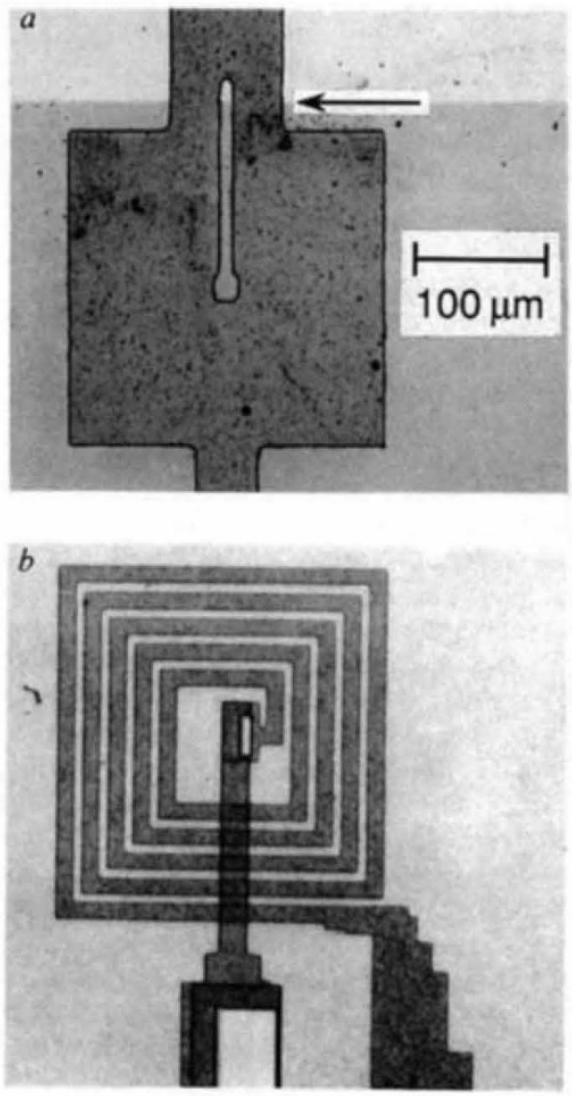

FIG. 1a, YBCO d.c. SOUID. The two Josephson junctions, formed by a $45^{\circ}$ grain boundary, are indicated with an arrow. $b$, Input coil of YBCO flux transformer. The two leads to the input coil are connected to a thin film pickup loop (not shown) with an area of 81 $\mathrm{mm}^{2}$. 